\title{
Image Processing Based Stiffness Mapping of a Haptic Device
}

\author{
B. Taner ${ }^{1}$, M. İ. C. Dede ${ }^{1}$ \\ ${ }^{1}$ Izmir Institute of Technology, Turkey,e-mail: baristaner@iyte.edu.tr, \\ candede@iyte.edu.tr
}

\begin{abstract}
.
The widely accepted performance criteria of haptic devices, which are transparency and z-width, are affected by the stiffness characteristics of the haptic device's mechanism. In addition indirect measurement of the handle pose of a haptic device is also affected by the stiffness characteristic. In this study, image processing techniques are used in the experimental setup to develop a stiffness map of a haptic device. The experimentally developed stiffness map is presented and the results are discussed by addressing future works.
\end{abstract}

Key words: Stiffness mapping, image processing, haptic device

\section{Introduction}

Haptic feedback in teleoperation systems provide users increased information about the distant site and enhances the feeling of being present in the remote site, which is generally termed as telepresence. During the haptic interaction, the interaction force and/or position information about the remote environment is provided to the user and also the haptic device allows the user to manipulate (by means of a telerobotic manipulation) the remote environment, which is called the slave environment in a teleoperation setting or virtual environment in virtual reality (VR) applications. In order to manipulate the slave, the pose of the haptic device's handle should be acquired. For precise operations, the motion tracking of the handle should be improved (Hirche \& Buss, 2012). These systems are human-in-the-loopsystems and the performance of the haptic system highly relies on the physical coupling and visuo-haptic co-location of the user's hand and the slave device in remote environment through a haptic interface (Barbieri et al., 2014).

Level of coupling of human and remote environment are dependent on properties of haptic devices in terms of transparency (LAWRANCE, 1992), (Hokayem $\&$ Spong, 2006) and impedance width or z-width (Colgate \& Brown, 1994). These properties, which are also called the performance criteria of haptic devices, are af- 
fected by several non-geometric inaccuracy factors; in particular, by the compliance of the haptic device mechanism. The effect of this inaccuracy factor on the performance of the haptic device can be reduced in preliminary design or in operation, using compensation techniques. Therefore, the compliance of the haptic devices must be calculated and/or obtained by using modeling by Finite Element Analysis (FEA), Structural Matrix Analysis (SMA), Virtual Joint Method (VJM) or by experimental methods that rely on absolute measurement of the compliant displacements of the haptic mechanism.

This paper describes procedure and results of obtaining the compliance mapping of a haptic device called HIPHAD v1.0, which is previously developed in IzTech Robotics Laboratory (Bilgincan et al., 2010), experimentally. A stereo camera system is used to reveal the compliant properties of this haptic device, which has an R-CUBE type translational mechanism. The novelty of the presented method is that cameras are used instead of interfering the mechanical systems impedance by additional hardware for measuring compliance matrix. Also, the stiffness properties of the haptic device HIPHAD are measured for the first time. This paper is organized so that the next section covers a brief description of the previously employed methods for calculating the stiffness properties of mechanisms. Later, the experimental test setup and camera calibration is explained. In the fourth section, the results of experimental compliant displacement measurements are given. Finally, the results are discussed and future works are addressed.

\section{Background Information}

In the previous works, modeling methods are formulated to obtain the stiffness matrix of mechanisms. Among these some provide relatively accurate results like the FEA methods and some provide computationally effective solution so that they can be used in on-line calculations. Although, the difference between the modeling-based results and experimental result are narrowing down with the improvements in the models, there is still a noteworthy error between these methods for high precision applications (Carbone, 2011). To identify the reason of deviation between the modeling and experimental methods, experimental methods can be studied. The previously conducted experiments share the same methodology of measuring the compliant displacement of the robot mechanism's end-effector under external forcing. In the literature, researchers have measured the end-effector displacements by various absolute measurement devices; for example, (Clinton, Zhang, \& Wavering, 1997) used dual indicators in measurement and followed the test procedure outlined in ASME Standard B5.54 (1993).

In another study, (Alici \& Shirinzadeh, 2005) made use of laser tracker for measuring the compliant displacement of a serial robot at heuristically determined points in its workspace. In this study, force is applied through a wire on the endeffector and resultant forces in three axes are measured via a force sensor. 
In the work of (Pinto, Corral, Altuzarra \& Hernández, 2010), a 5 step cycle procedure is defined for compliance measurements which is executed using inclinometer and a dial indicator; (1) dividing the workspace to nodes, (2) positioning the end-effector for each node, (3) preloading the end-effector, (4) measuring the initial position of the end-effector along each axis, (5) measuring the full loaded position of the end-effector along each axis.

Another method for measuring the compliant displacements is defined by (Ceccarelli \& Carbone, 2005), which they named it as Milli-CATRASYS. In this method, the compliant displacements measurement procedure initiates by acquiring the displacement information through the LVDT (Linear Variable Differential Transformers) sensors placed on the cables that are attached to the end-effector. Then, these displacements are used in calculating the end-effector's compliant displacements using the trilateration technique (Carbone, 2003). A possible problem with this measurement method is that the flexibility of the cables also play a part in the measurements. Therefore, observing the previous methods, our aim is to develop an experimental setup that can acquire the end-effector's compliant displacement without physically interacting with the mechanism. Vision-based absolute measuring system is an obvious solution, which is described next.

\section{Experimental Setup}

HIPHAD v1.0 was designed as a 6 degrees-of-freedom (DoF) kinesthetic haptic device that has a hybrid structure, which is composed of a 3 DoF active parallel translational mechanism, R-CUBE type, and a 3-DoF passive serial-spherical wrist mechanism. HIPHAD has a cubic workspace although R-CUBE mechanism has all active and passive revolute joints. More information on the mechanical design of the device can be found in (Bilgincan et al., 2010). Experimentation is executed by processing images taken by two cameras. Image processing algorithms used in this experimentation are run in Matlab.

Test rig is placed inside an enclosed dark room that is covering the surrounding of the test rig and LED light sources are used to provide uniform measurements with the vision system. The complete experimentation setup is presented in Fig. 1a with the numbers: (1) HIPHAD mechanism, (2) QUANSER Q8 data acquisition card (DAQ) for acquiring encoder measurements, (3) PC using the DAQ signals for calculating end-effector position indirectly by forward kinematics, (4) PC for processing images for stereo camera images to calculate the absolute position of the end-effector, (5) dark room, (6) LED strays, (7) one of the two cameras.

Stereo cameras are used for direct (absolute) measurement of the mobile platform / end-effector position. Therefore, the accuracy of the experimentation depends on the appropriate selection of these cameras. The lenses used in the cameras are FUJINON HF16HA-1B that have $16 \mathrm{~mm}$ focal length. Using this cameralens composition, smallest feature that can be detected by cameras is calculated to 
be at $0.165 \mathrm{~mm}$ for the camera axis that has 1600 pixels. A sub-pixel interpolation is implemented in vision algorithm to enhance the smallest feature that can be detected. To accommodate recalculation, radii of 4 circles that have different radii are measured using the measurement pattern. The selected diameters of the circles are $3.03,3.08,3.5,4 \mathrm{~mm}$. The diameter of the circle with $3.03 \mathrm{~mm}$ diameter is measured 20 times with the cameras and average of this measurement came out to be 68.2289 pixels with a standard deviation of $\sigma=0.0277$. For the circle having $3.08 \mathrm{~mm}$ diameter, image processing algorithm resulted in 70.2525 pixels for average diameter measurement with a standard deviation of $\sigma=0.0798251$. As a result, cameras along with the developed image processing algorithm found to be capable of measuring $0.025 \mathrm{~mm}$ with 1 pixel.

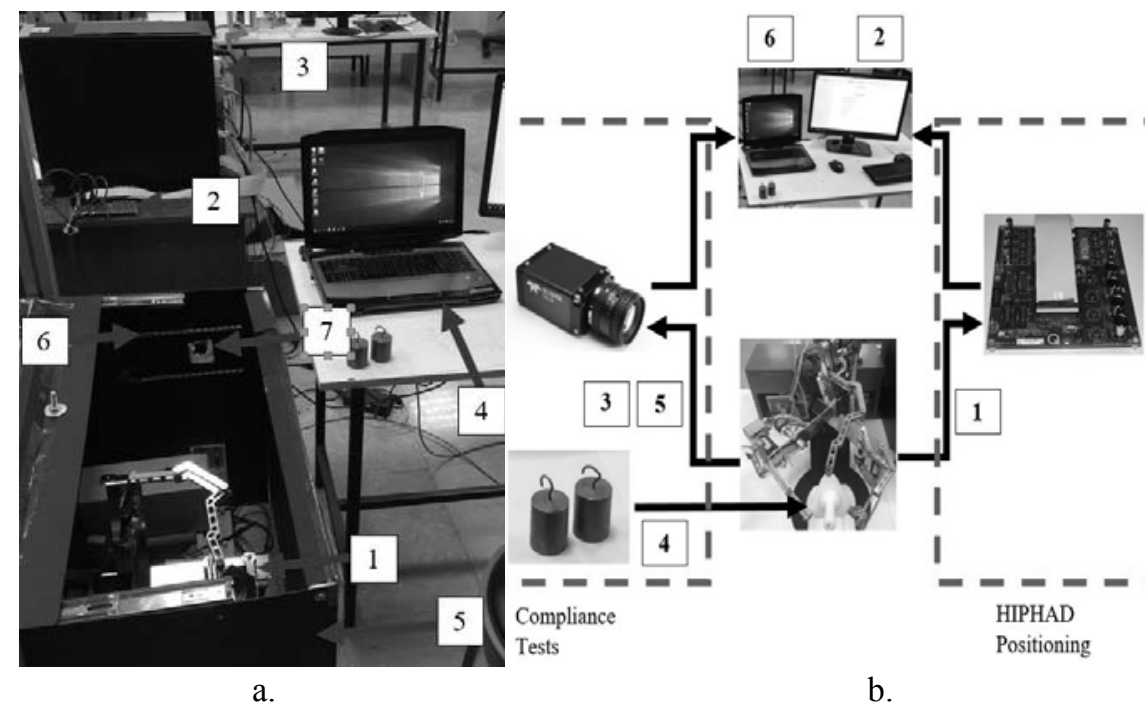

Fig. 1 Experimental test setup and procedure

\section{Experimentation Methodology}

The experiments are carried out by first determining the nodes to be used for measuring compliant displacements under external loads. The cubic workspace of the mechanism defined for the motion of the wrist point (in this case the endeffector) on the mobile platform is divided into three equal parts on each side of the cube resulting in 27 measurement nodes. To settle the wrist point of the HIPHAD on these specified nodes, the forward kinematics solution of HIPHAD is employed by using the acquired angular position values from encoders. It should be noted that the joint actuators are replaced with stoppers in order to retrain the 
motion of the first link rigidly. Whenever the HIPHAD mechanism is positioned on a specified node, the image acquisition process is initiated.

At this stage, 2 images are acquired which are taken before and after the force along the direction of measurement is applied on the mobile platform of HIPHAD. These images are processed by high pass filter and converted to a binary image. Following that, a blob analysis is applied on this binary image that is extracted from the raw image and coordinates of the tracking pattern in each position, which is the position of one of the calibration circles in initial and final positions. Then the positional difference is calculated and converted to world coordinate units in $\mathrm{mm}$. This testing procedure is schematically explained in Fig. 1b, in which the numbers denote the sequence of the stages. The sequence from 1 to 6 in Fig. $1 \mathrm{~b}$ is explained as follows: (1) measurement of the mobile platform's indirect position using encoders and acquiring the sensory data via Quanser Q8 DAQ, (2) position calculation of mobile platform by using forward kinematics to guarantee the settling of the mobile platform on the designated node, (3) image acquisition of the mobile platform when no external forces are applied, (4) application of external forces on the mobile platform by using calibrated weights, (5) image acquisition of the mobile platform under external forces, (6) image processing and compliant displacement calculation.

\section{Experimental Test Results}

Compliant displacement measurements are carried out when forces are applied along the Cartesian frame axes. The force along each axis is provided by pulling the mobile platform by a $700 \mathrm{~g}$ of calibrated mass that is passing through a plate with holes (to resemble the action of a pulley system), which is used to change the direction of the force. The calibrated mass provides forces in positive directions with respect to the world frame along $\mathrm{x}$ - and $\mathrm{y}$-axes. However, along $\mathrm{z}$-axis, the force created by the calibrated mass is in the negative direction. It should be noted that the compliant displacement measurements obtained at 9 nodes, which are on the same plane, are used in a cubic interpolation algorithm to estimate the compliant displacement values in between the nodes.

The workspace of the mechanism is placed in between $160 \mathrm{~mm}$ and $280 \mathrm{~mm}$ along each Cartesian axis. Test results for compliant displacement when the forces are applied along the $\mathrm{x}$-direction measured for the nodes on plane 1, which intersects y-axis at $160 \mathrm{~mm}$, yield the results provided in Fig. 2. In Fig. 2, 3 and 4, dark gray regions denote the workspace locations in which the mechanism has the highest stiffness on the respective plane and the light gray regions denote the workspace locations in which the mechanism has the highest compliance on the respective plane. In Fig. 2, the compliant displacement values range between 0.7 and $1.75 \mathrm{~mm}$. The maximum compliant displacement on this plane is found on node defined at $\mathrm{N}(160,280)$ by $1.72 \mathrm{~mm}$ and minimum compliant displacement is 
measured at node $\mathrm{N}(160,160)$ by $0.84 \mathrm{~mm}$ along the direction of the applied force, $(+) \mathrm{x}$-direction. The stiffness variations indicate that for plane 1, HIPHAD has a greater stiffness at the bottom corners and less stiffness at the top corners.

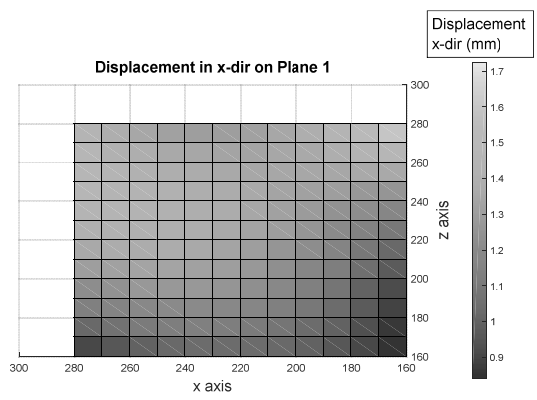

Fig. 2 Compliant displacement measurements of HIPHAD in x-axis on Plane 1.

On Plane 2, which intersects y-axis at $220 \mathrm{~mm}$, the compliant displacement values that are measured at the 9 nodes are presented in Fig. 3 in which the areas in between the nodes are calculated by cubic interpolation. Maximum and minimum displacement values on this plane are found on the nodes defined at $\mathrm{N}(280,280)$ and $\mathrm{N}(210,160)$, which are 2.56 and $1.33 \mathrm{~mm}$, respectively.

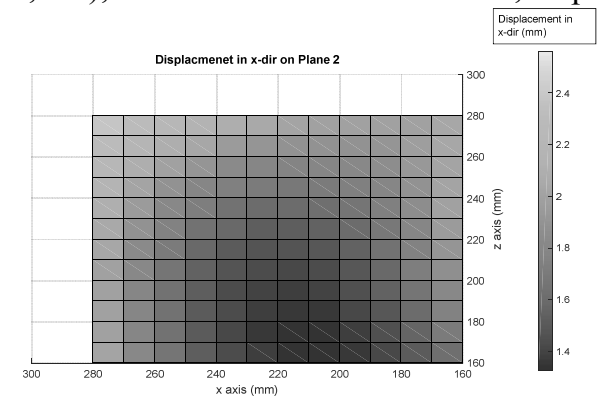

Fig. 3 Compliant displacement measurements of HIPHAD in x-axis on Plane 2.

Fig. 4 is plotted according to the compliant measurement data acquired on Plane 3, which intersects y-axis at $280 \mathrm{~mm}$. For this measurement set, maximum and minimum values of compliant displacements are measured on nodes $\mathrm{N}(280,280)$ and $\mathrm{N}(210,160)$, which came out to be 3.442 and $1.630 \mathrm{~mm}$, respectively. Similarity between the measurements on all planes is that the compliant displacements are increased towards the outer boundary of the workspace along zaxis, which was expected. 


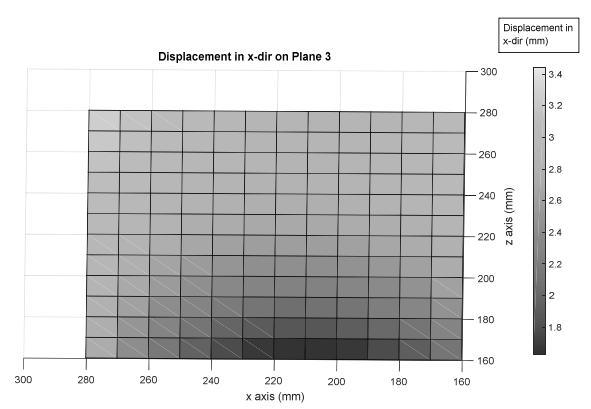

Fig. 4 Compliant displacement measurements of HIPHAD in x-axis on Plane 3.

To provide better visualization of HIPHAD compliance map, in between the nodes that measurements are received, interpolation is carried out for the whole workspace. It can be observed from Fig. 5 that HIPHAD displays an increasing compliance (or in other terms decreasing stiffness) values when the mobile platform is moved from the fully folded position at 160, 160 and $160 \mathrm{~mm}$ to the fully extended position at 280, 280 and $280 \mathrm{~mm}$ along $\mathrm{x}$, y and $\mathrm{z}$ directions, respectively. Figures $5 \mathrm{a}$ and $5 \mathrm{~b}$ show the same experiment results received for the external forces acting along $(+) \mathrm{x}$-axis in different views.

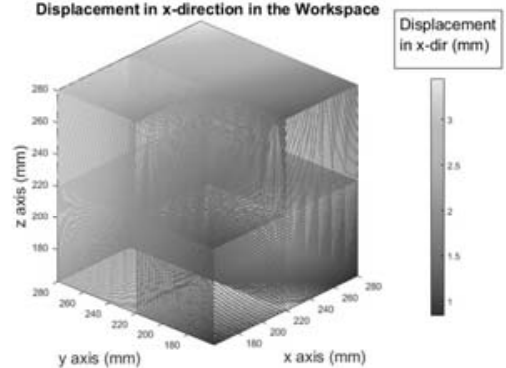

a

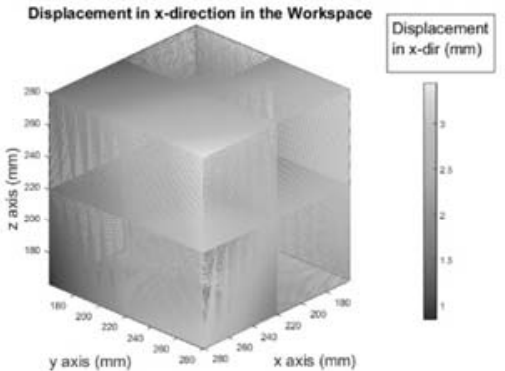

$\mathrm{b}$

Fig. 5 Compliant displacement map under $700 \mathrm{~g}$ force along $(+) \mathrm{x}$-axis throughout its workspace.

\section{Discussions and Conclusions}

In this study, stiffness characteristic of the HIPHAD haptic device is evaluated and mapped by using vision-based absolute position measurement method. The measurements are accommodated by using stereo cameras to capture absolute positions of the mobile platform and finding the difference between two cases; when no external load is applied and when external load is applied. It can be concluded from the results of this study that HIPHAD becomes more compliant on the most 
extended positions. However, it can be discussed that the measured displacement of the mobile platform under external forces is not only a result of the compliant behavior of the mechanism but joint wear and joint clearance also play a part.

Based on the obtained stiffness characteristics of HIPHAD, future work on the design changes for the HIPHAD mechanism can include an improved joint structure design that has smaller joint clearances and use of materials with high strength-to-weight ratio, such as carbon-fiber pipes, for constructing the links. Another future work is to obtain a computationally effective and relatively accurate stiffness model of HIPHAD to be used in increasing the precision in the handle's motion acquisition under the influence of the forces applied by the human.

\section{Acknowledgements}

This work is supported in part by The Scientific and Technological Research Council of Turkey via grant number 115E726.

\section{References}

1. Alici, G. \& Shirinzadeh, B. (2005). Enhanced stiffness modeling, identification and characterization for robot manipulators. Robotics, IEEE Transactions on, 21(4), 554-564.

2. Barbieri, L., Bruno, F., Cosco, F. \& Muzzupappa, M. (2014). Effects of device obtrusion and tool-hand misalignment on user performance and stiffness perception in visuo-haptic mixed reality. International Journal of Human-Computer Studies, 72(12), 846-859.

3. Bilgincan T., Gezgin E., and Dede M. I. C., "Integration of the Hybrid-Structure Haptic Interface HIPHAD v1.0,": Proceedings of the International Symposium of Mechanism and Machine Theory, Izmir, Turkey, pp. 267-284, October 5-8, 2010.

4. Carbone, G. (2011). Stiffness analysis and experimental validation of robotic systems. Frontiers of Mechanical Engineering, 6(2), 182-196.

5. Ceccarelli, M. \& Carbone, G. (2002). A stiffness analysis for CaPaMan (Cassino parallel manipulator). Mechanism and Machine Theory, 37(5), 427-439.

6. Clinton, C. M., Zhang, G. \& Wavering, A. J. (1997). Stiffness Modeling of a Stewart Platform Based Milling Machine.

7. Colgate, J. E. \& Brown, J. M. (1994, May). Factors affecting the z-width of a haptic display. In Robotics and Automation, 1994. Proceedings, 1994 IEEE International Conference on (pp. 3205-3210). IEEE.

8. Hirche, S. \& Buss, M. (2012). Human-oriented control for haptic teleoperation. Proceedings of the IEEE, 100(3), 623-647.

9. Hokayem, P. F. \& Spong, M. W. (2006). Bilateral teleoperation: An historical survey. Automatica, 42(12), 2035-2057.

10. D. A. Lawrence, "Stability and transparency in bilateral teleoperation," in IEEE Transactions on Robotics and Automation, vol. 9, no. 5, pp. 624-637, Oct 1993. doi: 10.1109/70.258054

11. Pinto, C., Corral, J., Altuzarra, O. \& Hernández, A. (2010). A methodology for static stiffness mapping in lower mobility parallel manipulators with decoupled motions. Robotica, 28(05), 719-735. 